\title{
Enhanced differentiation of retinal progenitor cells using microfabricated topographical cues
}

\author{
Mark R. Steedman • Sarah L. Tao • Henry Klassen • \\ Tejal A. Desai
}

Published online: 14 January 2010

(C) The Author(s) 2010. This article is published with open access at Springerlink.com

\begin{abstract}
Due to the retina's inability to replace photoreceptors lost during retinal degeneration, significant interest has been placed in methods to implant replacement cells. Polymer scaffolds are increasingly being studied as vehicles for cellular delivery to degenerated retinas. Previously, we fabricated poly(methyl methacrylate) thin film scaffolds that increased survival and integration of implanted retinal progenitor cells (RPCs). Additionally, these scaffolds minimized the trauma and cellular response associated with implantation of foreign bodies into mouse eyes. Here, we demonstrate that biodegradable polycaprolactone (PCL) thin film scaffolds can be fabricated with integrated microtopography. Microfabricated topography in a PCL thin film enhanced the attachment and organization of RPCs compared to unstructured surfaces. Using realtime quantitative polymerase chain reaction we also observed that attachment to microtopography induced
\end{abstract}

M. R. Steedman • T. A. Desai

University of California San Francisco/University of California Berkeley Joint Graduate Group in Bioengineering,

San Francisco, CA, USA

M. R. Steedman · T. A. Desai $(\square)$

Department of Bioengineering and Therapeutic Sciences and Department of Physiology,

University of California San Francisco,

San Francisco, CA 94158-2330, USA

e-mail: Tejal.Desai@ucsf.edu

S. L. Tao

The Charles Stark Draper Laboratory, Inc.,

Cambridge, MA, USA

H. Klassen

Gavin Herbert Eye Institute, Department of Ophthalmology,

University of California Irvine,

Orange, CA, USA cellular differentiation. RPCs grown on PCL thin films exhibited an increase in gene expression for the photoreceptor markers recoverin and rhodopsin, an increase in the glial and Müller cell marker GFAP, and a decrease in SOX2 gene expression (a marker for undifferentiated progenitor cells) compared to cells grown on unmodified tissue culture polystyrene (TCPS).

Keywords Microfabrication - Retina $\cdot$ Tissue engineering · Polycaprolactone $\cdot$ Macular degeneration

\section{Introduction}

Degenerative diseases of the retina, including retinitis pigmentosa and age-related macular degeneration, are characterized by photoreceptor loss and eventually lead to irreversible visual disability (Abdelsalam et al. 1999; Rivolta et al. 2002; Kulkarni and Kuppermann 2005; Hartong et al. 2006). Progress of retinal degeneration is typically slow, and many current clinical treatments focus primarily on delaying disease progression (Delyfer et al. 2004; Bhisitkul 2006; Bashshur et al. 2007). In the advanced stages of the diseases, photoreceptors are irreversibly lost due to the minimal innate ability of the eye to regenerate damaged tissue. To overcome this problem, a promising technique has emerged whereby an exogenous source of photoreceptors is introduced into the subretinal space (Meyer 2006).

During retinal degeneration, retinal circuitry initially remains intact, and implanted cells only need to make short synaptic connections to host tissue. Implanted multipotent retinal progenitor cells (RPCs) have been shown to migrate through a degenerating retina, extend processes as far as the optic nerve, and express markers of mature retinal neurons 
and glial cells (Takahashi et al. 1998; Young, et al. 2000; Klassen, et al. 2004b; Meyer et al. 2006; Canola et al. 2007; Klassen et al. 2007). Additionally, RPCs committed to a photoreceptor cell fate integrate, differentiate, form synaptic connections, and improve visual function upon implantation (MacLaren et al. 2006). Despite these successes, bolus injections of cells to the subretinal space led to a high degree of cell death (Tomita et al. 2005). Minimizing cell death during implantation and increasing the number of viable cells implanted are subsequently areas for significant improvement of retinal tissue engineering.

Polymer scaffolds have been utilized in recent years to increase the number of viable cells delivered to a degenerated retina (Lavik et al. 2005; Tomita et al. 2005; Tao et al. 2007; Neeley et al. 2008; Redenti et al. 2009). First generation polymer scaffolds were fabricated from poly(L-lactic acid)/poly(lactic-co-glycolic acid) (PLLA/ PLGA) with a ten-fold increase in cell delivery (Tomita et al. 2005). However, significant complications resulted from implantation including: inflammation, fibrosis, significant foreign body response (Sundback et al. 2005), and some cases led to retinal detachment due to scaffold thickness $(\sim 250 \mu \mathrm{m})$ (Lavik et al. 2005; Tomita et al. 2005; Warfvinge et al. 2005). Previously, we showed that a spin-cast poly(methyl methacrylate) (PMMA) thin film scaffold $(6 \mu \mathrm{m})$ reduced the occurrence of trauma during implantation, and implanted RPCs were able to express at least three markers of mature retinal cells (Tao et al. 2007). The limitation of a PMMA scaffold is that it is nondegradable and remains in the subretinal space permanently or until removed. A biodegradable scaffold made of poly (glycerol-sebacate) (PGS) of intermediate thickness (45 $\mu \mathrm{m})$ has been shown to retain large amounts of RPCs through simulated implantations (Neeley et al. 2008; Redenti et al. 2009).

Another potential scaffold material is polycaprolactone (PCL), which has been previously applied extensively for fabrication of medical implants (Lowry et al. 1997; Huang et al. 2002; Giavaresi et al. 2004; Holmbom et al. 2005; Chiari et al. 2006) including subretinal steroid delivery (Beeley et al. 2005). It is biodegradable, biocompatible, and can be spin-cast into a thin film (Tang et al. 2007). Previously, we showed that a structured PCL thin film scaffold $(5 \mu \mathrm{m})$ increased expression of mature retinal markers (Redenti et al. 2008). Furthermore, PCL-delivered RPCs migrated into both normal and degenerated retina, demonstrating it may be an ideal material for retinal tissue regeneration.

In addition to delivering an appropriate number of healthy cells to the retina, a polymer scaffold might also promote differentiation of implanted cells prior to implantation to improve the yield of desired cellular phenotypes and decrease the probability of undesired mitotic activity post-implantation. PLLA/PLGA and PGS scaffolds have demonstrated the ability to promote differentiation of RPCs in vitro prior to implantation. This was established by associating cell attachment of the non-adherent RPCs to the polymer scaffolds with changes in gene expression (Lavik et al. 2005; Tomita et al. 2005; Neeley et al. 2008; Redenti et al. 2009). Other studies have also shown that cell attachment to polymer membranes can induce differentiation, including human bone marrow-derived mesenchymal progenitor cells (Endres et al. 2003), human marrow stromal cells (Marletta et al. 2007), and human chondrocytes (Garcia-Giralt et al. 2008). However, this can be more difficult for cell types such as RPCs which are grown in serum-free media supplemented with epidermal growth factor (EGF), and often form non-adherent neurospheres. Although RPCs proliferate rapidly under these conditions, they do not readily adhere to a growth substrate, such as tissue culture polystyrene (TCPS), unless it has been treated with an extracellular matrix protein, such as laminin (Klassen et al. 2004b), a component of serum. Furthermore, RPCs cultured with serum without supplemental EGF lose their ability to proliferate and attach to the TCPS surface (Klassen et al. 2004a; Tomita et al. 2005). Some RPCs grown in these conditions differentiated and expressed mature retinal cell markers such as the Müller glial cell marker glial fibrillary acidic protein (GFAP), the rod bipolar cell marker protein kinase $\mathrm{C}$ alpha $(\mathrm{PKC}-\alpha)$, and the rod photoreceptor markers recoverin and rhodopsin (Klassen et al. 2004a; Tomita et al. 2005). Ideally a scaffold material would not require any surface modification to induce cell attachment. Previous scaffolds made from PGS (Neeley et al. 2008; Redenti et al. 2009) and PMMA (Tao et al. 2007) required protein modification of the surface with either laminin or a combination of laminin and poly-Llysine to achieve RPC attachment. Additionally, PLLA/ PLGA scaffolds needed a concentrated cell solution to be added to the scaffolds drop-wise, which was then incubated overnight before more media was added (Lavik et al. 2005).

Recently, the physical extracellular environment has increasingly been investigated to elucidate cell-substrate interactions. Several studies have shown that microscale and nanoscale topography induce reorientation and differentiation for multiple cell types, including astroglial cells (Recknor et al. 2004), smooth muscle cells (Yim et al. 2005), mesenchymal stem cells (Yim et al. 2007), neural progenitor cells (Recknor et al. 2006), human corneal epithelial cells (Karuri et al. 2008), and bovine aortic endothelial cells (Wang et al. 2007). In particular, a number of studies have demonstrated the use of soft lithography to create topographical features with polydimethylsiloxane (PDMS), as this substrate is inexpensive, nontoxic, easily fabricated, and rapidly prototyped (Whitesides et al. 2001; Motlagh et al. 2003; Thakar et al. 2008). For tissue 
engineering applications, however, PDMS is an inadequate substrate, as it does not degrade. Therefore, a material that is biodegradable, biocompatible, can be spin cast into thin films, and patterned to create microtopographical features would be valuable for tissue engineering applications. Here, we have developed a method to fabricate $5 \mu \mathrm{m}$ thick degradable PCL thin films that maintain an intended structural micro-architecture. Additionally, we demonstrate that specific microtopographical cues can enhance RPC attachment and differentiation in vitro using quantitative polymerase chain reaction.

\section{Materials and methods}

\subsection{Polycaprolactone thin film scaffold fabrication}

Polycaprolactone thin films were fabricated using a threestep process (Fig. 1(a)). First, a micropatterned silicon master was fabricated using standard photolithography techniques (Duffy et al. 1998). A layer of SU-8 2010 negative photoresist (Microchem, Newton, MA) was spin cast at $1000 \mathrm{rpm}$ for $30 \mathrm{sec}$ onto a 3" silicon wafer (Addison Engineering, San Jose, CA) using a PMW32 spin coater (Headway Research, Garland, TX) and pre-baked at $95^{\circ} \mathrm{C}$ for $3.5 \mathrm{~min}$. An array of microwells was then

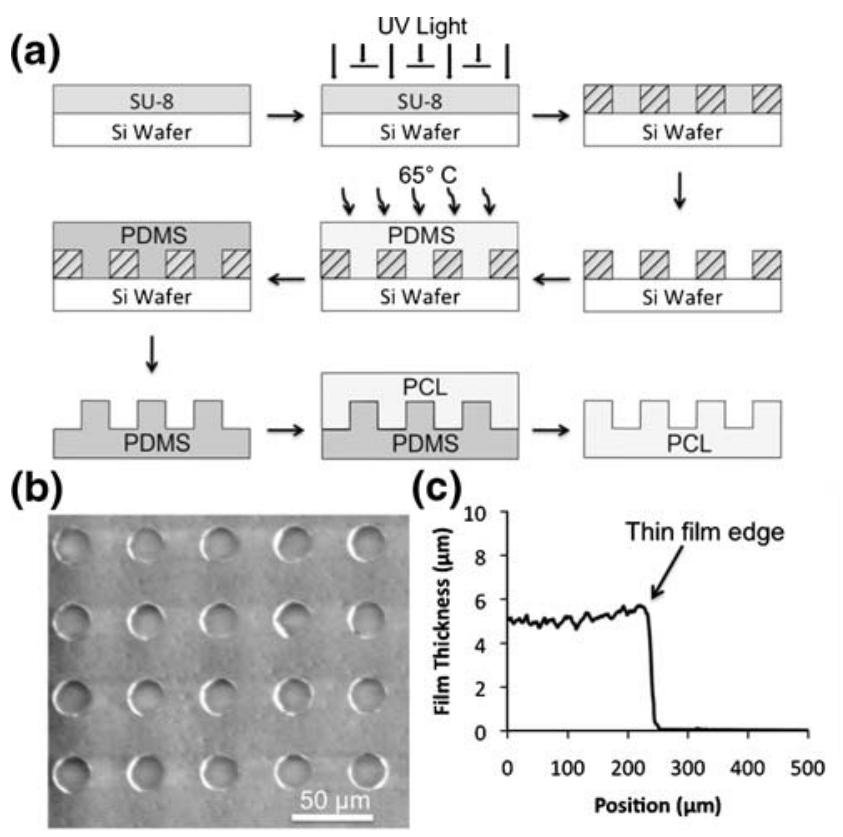

Fig. 1 (a) Schematic of PCL thin film scaffold fabrication. SU8 photoresist is spin-cast onto a silicon wafer and exposed to UV light through a negative mask. Unexposed areas are not crosslinked and developed away, and PDMS is cured on the wafer. After peeling the PDMS mold from the wafer, PCL is spin-cast on the mold and peeled from the surface. (b) A scanning electron micrograph of a PCL thin film with $25 \mu \mathrm{m}$ diameter wells. (c) Profile of PCL thin film patterned into the photoresist using a photomask and exposing the photoresist to UV light for $30 \mathrm{sec}$ at an intensity of $5 \mathrm{~mW} \mathrm{~cm}{ }^{-2}$ using a Karl Suss MJB 3 mask aligner (SUSS MicroTec Inc., Waterbury Center, VT). The SU-8 molds were then post-baked at $95^{\circ} \mathrm{C}$ for $4.5 \mathrm{~min}$ and developed with SU-8 Developer (Microchem, Newton, MA) for $2 \mathrm{~min}$. Second, an inverse pattern of the silicon wafer was fabricated using polydimethylsiloxane (Sylgard 184, Dow Corning, Midland, MI). The base and curing agent were mixed at a 10:1 (v:v) ratio, degassed under vacuum, poured onto the SU-8 micropatterned wafer, and baked at $65^{\circ} \mathrm{C}$ for $2 \mathrm{~h}$. Once cured, the PDMS was peeled from the silicon master. Third, a polymeric thin film containing the inverse pattern of the PDMS was fabricated by spin casting a solution of polycaprolactone (MW 80,000 , Sigma-Aldrich, St. Louis, MO) on top of the PDMS. Polycaprolactone was dissolved in 2,2,2-trifluoroethanol (Sigma-Aldrich, St. Louis, MO) at $65^{\circ} \mathrm{C}$ for $3 \mathrm{~h}$ at a concentration of $0.1 \mathrm{~g} \mathrm{~mL}^{-1}$. Polycaprolactone solution was poured onto the micropatterned PDMS and spin coated at $1500 \mathrm{RPM}$ for $1 \mathrm{~min}$, after which the polycaprolactone thin film was detached and peeled from the PDMS using forceps. Prior to cell seeding, PCL thin films were sterilized via exposure to UV light for $1 \mathrm{~h}$.

\subsection{Profilometry}

Characterization of PCL thickness was conducted with an Ambios Technology XP-2 Stylus Profiler (Santa Cruz, CA). The profilometry image was achieved using a scan speed of $0.03 \mathrm{~mm} \mathrm{sec}$, a length of $0.5 \mathrm{~mm}$ and a stylus force of $0.5 \mathrm{mg}$.

\subsection{Mouse retinal progenitor cells}

Retinal progenitor cells were isolated and maintained as described previously (Okabe et al. 1997; Klassen et al. 2004b). Briefly, cells were cultured in NeuroBasal medium (NB) (Invitrogen, Carlsbad, CA) supplemented with $2 \mathrm{mM}$ L-Glutamine (Sigma-Aldrich, St. Louis, MO), 100 $\mu \mathrm{g} \mathrm{mL}{ }^{-1}$ penicillin-streptomycin (Sigma-Aldrich, St. Louis, MO), $20 \mathrm{ng} \mathrm{mL}^{-1}$ epidermal growth factor (EGF; Promega, Madison, WI), and neural supplement (B27; Invitrogen, Carlsbad, CA) and plated into T-75 culture flasks as nonadherent neurospheres. On alternating days, half of the cell solution was removed from the culture flask and centrifuged at $1000 \mathrm{RPM}$ for $3 \mathrm{~min}$. The media was removed and the pellet of cells was re-suspended in fresh medium and returned to the flask. Cells were passaged 1:10 every 7 days. All cells used in this study were passaged fewer than 15 times. For all experiments, cells were seeded at an initial density of 5000 cells $\mathrm{mm}^{-2}$ and grown for 2 days. 


\subsection{Cell Attachment}

Thin films were immobilized in custom-made holders and fitted into a 12-well plate. After 2 days of growth cells were fixed in $4 \%$ formaldehyde for $2 \mathrm{~h}$, rinsed twice with PBS, and then permeabilized with $0.25 \%$ Triton $\mathrm{X}-100$ for $1 \mathrm{~h}$. Nuclei were stained with DAPI and mounted between a glass slide and coverslip using SlowFade Gold with DAPI (Invitrogen, Carlsbad, CA). A $3.7 \mathrm{~mm}^{2}$ area of the thin film was imaged using an Olympus BX60 Microscope (Center Valley, PA), and the number of cells on each surface was manually quantified. Figure 2 represents data from three individual experiments.

\subsection{Scanning electron microscopy}

Cells were fixed in a solution of 3\% glutaraldehyde (Sigma-Aldrich, St. Louis, MO), 0.1 M sucrose (SigmaAldrich, St. Louis, MO), and $0.1 \mathrm{M}$ sodium cacodylate (Sigma-Aldrich, St. Louis, MO) buffer for $72 \mathrm{~h}$ at room temperature. Following fixation, samples were rinsed in buffer containing $0.1 \mathrm{M}$ sucrose and $0.1 \mathrm{M}$ sodium cacodylate for $5 \mathrm{~min}$. Samples were then dehydrated by removing the sucrose-cacodylate buffer and adding a series of aqueous ethanol solutions for $10 \mathrm{~min}$ each in a graded series as follows: 35\%, 50\%, 70\%, 95\%, 100\% (twice). The final $100 \%$ ethanol solution was replaced with hexamethyldisilazane (HMDS) (PolySciences, Inc., Warrington, PA) for $10 \mathrm{~min}$ and removed. Samples were (a)

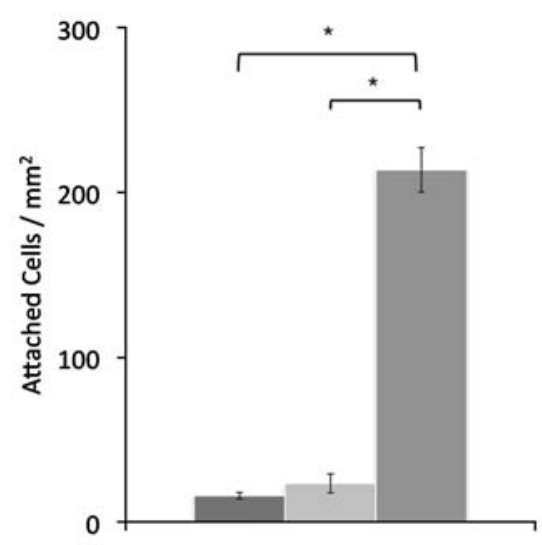

" TCPS $=$ Unstructured PCL $=25 \mu \mathrm{m}$ well PCL (b)

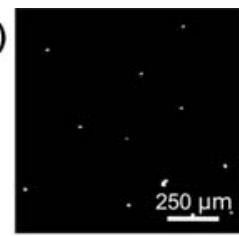

(c)

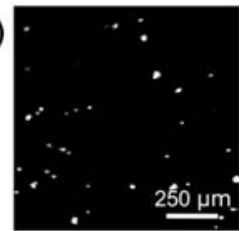

(d)

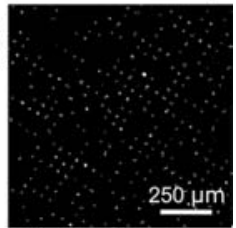

Fig. 2 (a) Attachment of RPCs to substrate surfaces after 2 days growth. Substrate microtopography of $25 \mu \mathrm{m}$ well PCL leads to significantly more RPC attachment compared to unstructured PCL and TCPS surfaces. Fluorescence images of DAPI-stained RPC nuclei attached to (b) TCPS, (c) unstructured PCL, and (d) $25 \mu \mathrm{m}$ well PCL. $* p<0.05$, Student-Newman, Keuls test. Error bars indicate standard deviation over three independent experiments allowed to air dry for $30 \mathrm{~min}$ before mounting and imaging. Samples were imaged using a NovelX mySEM (Lafayette, CA) scanning electron microscope with an accelerating voltage of $1 \mathrm{kV}$.

\subsection{Real-time quantitative RT-PCR (qPCR)}

Messenger RNA levels were quantified using a Fast SYBR $^{(\mathbb{B}}$ Green Cells-to-CT ${ }^{\mathrm{TM}}$ Kit (Applied Biosystems, Foster City, CA). Reverse transcription was performed on a Mastercycler ep gradient S (Eppendorf, Hamburg, Germany). Real-time quantitative PCR was performed using a StepOne Plus (Applied Biosystems, Foster City, $\mathrm{CA}$ ) run for 40 cycles. The primers (Integrated DNA Technologies, San Diego, CA) used in this study are shown in Table 1. Each reaction was performed in triplicate and normalized to glyceraldehyde 3-phosphate dehydrogenase (GAPDH) as an endogenous control.

\section{Results}

\subsection{Thin film fabrication}

Dissolved PCL was spin-cast onto a PDMS mold with an array of $25 \mu \mathrm{m}$ diameter micropegs, resulting in a thin layer of polymer coating the PDMS surface. Flexibility of the PDMS mold allowed the PCL to be peeled from the surface, producing a thin film with $25 \mu \mathrm{m}$ diameter microwells (an inverse of the PDMS pattern) (Fig. 1(a) and (b)). PCL films were also fabricated from an unstructured PDMS mold and used as a control for potential material effects on cellular differentiation. Film thickness is dependent on the spin speed and viscosity of the dissolved polymer solution and was measured to be 5 $\mu \mathrm{m}$ thick (Fig. 1(c)).

Table 1 qPCR Primers

\begin{tabular}{ll}
\hline Gene & Primer Sequence $\left(5^{\prime}-3^{\prime}\right)$ \\
\hline Recoverin & F: TGTGAAACTCCTCCCAGATGATG \\
& R: TTCCAAAGAATGCCCAGATCTT \\
Rhodopsin & F: TTATGTGCCCTTCTCCAACGT \\
& R: TGGTTCCGCCAGGTAGTACGT \\
GFAP & F: CAGCCCTGAAGAATCCACAAC \\
& R: CCCTTAGCTTGGAGAGCAACA \\
SOX2 & F: TTCCAAAAACTAATCACAACAATCG \\
& R: GAGACGGGCGAAGTGCAA \\
GAPDH & F: TGGCCTCCAAGGAGTAAGAAAC \\
& R: GGGATAGGGCCTCTCTTGCT \\
\hline
\end{tabular}


Fig. 3 Scanning electron micrographs of RPCs grown on (a) TCPS, (b) unstructured PCL, and (c) PCL with $25 \mu \mathrm{m}$ diameter wells. RPCs retain a circular morphology regardless of the substrate surface but do not form clusters on either PCL surface
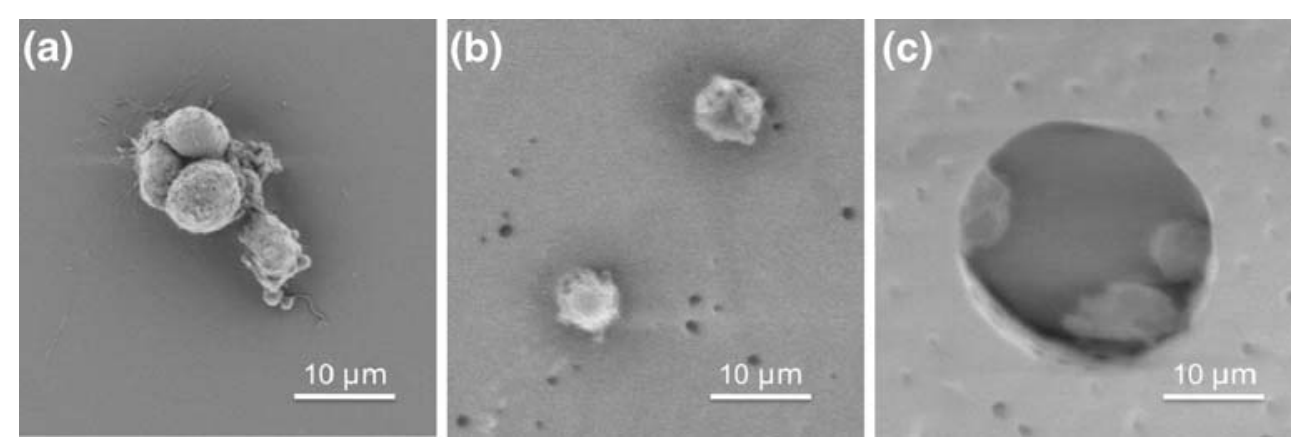

\subsection{RPC attachment to thin film PCL scaffolds}

RPCs were seeded on thin film scaffolds and TCPS at an initial density of 5000 cells $\mathrm{mm}^{-2}$. A small number of cells $\left(<30\right.$ cells $\left.\mathrm{mm}^{-2}\right)$ attached to the two unstructured surfaces (Fig. 2), either as small clusters (Fig. 3(a)) or as individual cells (Fig. 3(b)), although the vast majority of cells remained in solution as non-adherent neurospheres (Fig. 3). In contrast, RPC attachment to PCL with $25 \mu \mathrm{m}$ diameter wells resulted in a ten-fold increase in cell attachment relative to unstructured substrates (Fig. 2). Cells adhered to the inside of microwells, usually either on the edges or the bottom of wells, with as many as three or four cells in each well (Fig. 3(c)).

\subsection{Cell morphology}

Cells on each substrate maintained a similar circular morphology; however, RPCs grown on TCPS formed clusters, while cells grown on both PCL substrates attached as individual cells, even when found in close proximity to each other, such as in the event of being confined inside a microwell (Fig. 3(c)). It is possible that cell-cell communication is paramount to RPC proliferation, and therefore the failure of RPCs to re-cluster on PCL substrates induces differentiation. To investigate this, gene regulation of differentiation markers was determined using quantitative real-time polymerase chain reaction (qPCR).

\subsection{Quantitative real-time polymerase chain reaction}

Analogous to the attachment experiments, RPCs were grown under normal proliferative conditions on the same three substrates, and mRNA expression levels were quantified using qPCR. Four gene targets were investigated using the primers shown in Table 1. These included genes for recoverin, rhodopsin, glial fibrillary acidic protein (GFAP), and SOX2. Recoverin is a calcium-binding protein found in photoreceptors, rhodopsin is a G-protein coupled receptor also found in photoreceptors, and GFAP is an astrocyte marker, all three being markers for differentiation. SOX2 is also included as a marker for undifferentiated proliferating cells. Figure 4 shows the ratio of mRNA expression of each target gene to glyceraldehyde 3phosphate dehydrogenase (GAPDH), a housekeeping gene commonly used to account for cell number variation. All three differentiation markers were up-regulated for both PCL surfaces, and SOX2 was down-regulated on the two PCL surfaces relative to TCPS. Additionally, up-regulation of recoverin and rhodopsin was significantly higher on films with microtopography compared to unstructured PCL, suggesting that topographical cues not only enhance attachment but also RPC differentiation.

\section{Discussion}

The ability to fabricate thin films is paramount to the success of retinal tissue engineering. Initial attempts at injecting RPCs into the subretinal space resulted in death for nearly ninety percent of the grafted cells (Tomita et al. 2005). To circumvent this problem, new vehicles for cell delivery were explored. Previously, polymer scaffolds were implanted into the subretinal space of animal models to avoid damaging the outer retina, although the use of thicker scaffolds still resulted in trauma to the host. Minimizing the

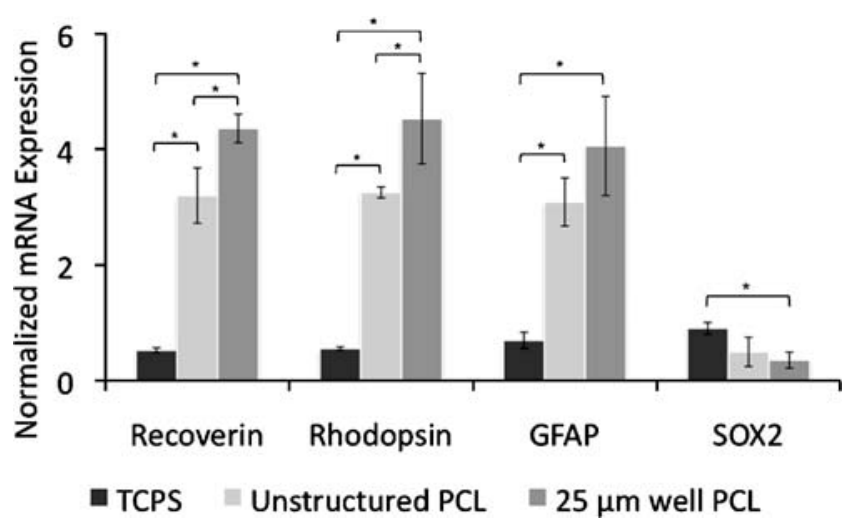

Fig. 4 mRNA expression normalized to GAPDH for RPCs grown in proliferative conditions. Expression of rod photoreceptor markers recoverin and rhodopsin and glial cell marker GFAP are increased on unstructured PCL and PCL with $25 \mu \mathrm{m}$ diameter wells compared to TCPS. ${ }^{*} p<0.05$, Student-Newman, Keuls test. Error bars indicate standard deviation over three independent experiments 
thickness of an implant, especially in the subretinal space, limits the potential damage that can occur during and after implantation. The PCL thin film scaffolds used in this study ( $5 \mu \mathrm{m}$ thickness) are fifty times thinner than the original PLLA/PLGA scaffolds (Lavik et al. 2005; Tomita et al. 2005) used and nine times thinner than the more recent PGS scaffolds (Neeley et al. 2008; Redenti et al. 2009).

Another critical aspect of retinal tissue engineering is cell attachment to the scaffold surface. To successfully implant RPCs into the subretinal space, cells must be firmly attached to the scaffold surface and be able to resist shear forces that occur during implantation of the film. Previous studies have indicated that scaffold topography can increase progenitor cell attachment, although protein modification of the surface has been used to induce cell attachment and differentiation (Recknor et al. 2006; Tao et al. 2007). Here, to determine if either the polymer material or microtopography could potentially induce the same behavior of attachment, RPCs were seeded on PCL in the absence of chemical or protein modification. RPC attachment to the scaffold microtopography resulted in a highly organized pattern of cells (Fig. 2(d)). The microwell topography also shielded attached cells from shear forces present in processes, such as staining and SEM preparation, and presumably would provide the same effect during implantation.

Cell attachment to unstructured and microstructured PCL resulted in the absence of clustering found in proliferating RPCs, although no further dramatic morphological changes occurred. It is clear the presence of microtopography influenced both cell attachment and gene expression. The up-regulation of fate-specific markers such as recoverin, rhodopsin, and GFAP indicates enhanced differentiation of the RPCs toward rod and glial cell fates, which is confirmed by the down-regulation of the proliferation marker SOX2. For the purpose of treating retinal degeneration, RPCs would ideally differentiate into rod photoreceptors. The cells used in this study have earlier been shown to primarily produce rod, bipolar, and glial cells, (Redenti et al. 2008) and it is therefore possible microtopographical cues increase the rate of differentiation of individual RPCs into a predetermined differentiation pathway. Future studies that elucidate individual cell fates rather than global trends would therefore be valuable. It is also possible chemical cues in conjunction with microtopography may limit differentiation into a glial cell fate and promote rod specific differentiation.

In this study we demonstrate for the first time that microscale topographical cues influence cell attachment and differentiation of retinal progenitor cells independent of any biochemical cues. Interestingly, although RPC attachment to PCL microwells resulted in up-regulation of fate specific markers, such as recoverin, rhodopsin, and GFAP, no dramatic change in morphology was observed. This is in contrast to a previous study in which RPCs were grown on PCL thin films with a nanostructured surface (Redenti et al. 2008). As evidenced from this and other studies, it may be possible to generate fully differentiated photoreceptors from RPCs for implantation using a combination of physical and biochemical cues. Further studies involving prolonged growth of RPCs on microstructured PCL and in vivo implantations of PCL thin films would be beneficial in determining the full extent to which microtopography influences progenitor cell fate determination.

\section{Conclusions}

In this study we have shown that microscale topography can strongly influence attachment and differentiation of retinal progenitor cells independent of any biochemical cues. Using comparative analysis of gene expression levels by qPCR, we have shown that RPCs grown on microfabricated topographical features express higher levels of photoreceptor and glial cell markers compared to RPCs grown on unstructured surfaces. Additionally, the thin film PCL scaffolds used in this study are on the order of the same thickness of other PMMA and PCL scaffolds that have been used with minimal trauma to the host. These results indicate that substrate topography influences RPC differentiation, and a biodegradable PCL thin film scaffold is a promising material for delivering RPCs to degenerated retinas.

Acknowledgements All microfabrication work was performed in the UCSF Micro- and Nanofabrication Core. We thank Dr. Kristy M. Ainslie, Dr. Daniel A. Bernards, Dr. Rachel D. Lowe, Alana G. Lerner, and Lily Peng for their valuable insight and advice. This research was supported by a NSF NSEC grant to T.A.D., a grant from the California Institute for Quantitative Biosciences (QB3) to T.A.D., a grant from the Lincy Foundation to H.K., a Sandler Family Foundation Translational Research Fellowship to S.L.T., a Knights Templar Eye Foundation Investigator Grant to S.L.T., and a grant from the Foundation Fighting Blindness to S.L.T.

Open Access This article is distributed under the terms of the Creative Commons Attribution Noncommercial License which permits any noncommercial use, distribution, and reproduction in any medium, provided the original author(s) and source are credited.

\section{References}

A. Abdelsalam, L. Del Priore et al., Drusen in age-related macular degeneration: Pathogenesis, natural course, and laser photocoagulation-induced regression. Surv Ophthalmol 44(1), $1-29$ (1999)

Z.F. Bashshur, A. Schakal et al., Intravitreal bevacizumab vs verteporfin photodynamic therapy for Neovascular age-related macular degeneration. Arch Ophthalmol 125(10), 1357-1361 (2007) 
N.R. Beeley, J.V. Rossi et al., Fabrication, implantation, elution, and retrieval of a steroid-loaded polycaprolactone subretinal implant. J Biomed Mater Res A 73(4), 437-44 (2005)

R.B. Bhisitkul, Vascular endothelial growth factor biology: clinical implications for ocular treatments. Br J Ophthalmol 90(12), 1542-1547 (2006)

K. Canola, B. Angenieux et al., Retinal stem cells transplanted into models of late stages of retinitis pigmentosa preferentially adopt a glial or a retinal ganglion cell fate. Investig. Ophthalmol. Vis. Sci. 48(1), 446-454 (2007)

C. Chiari, U. Koller et al., A tissue engineering approach to meniscus regeneration in a sheep model. Osteoarthritis Cartilage 14(10), 1056-1065 (2006)

M.N. Delyfer, T. Leveillard et al., Inherited retinal degenerations: therapeutic prospects. Biol Cell 96(4), 261-269 (2004)

D.C. Duffy, J.C. McDonald et al., Rapid prototyping of microfluidic systems in poly(dimethylsiloxane). Anal Chem 70(23), 4974 4984 (1998)

M. Endres, D.W. Hutmacher et al., Osteogenic induction of human bone marrow-derived mesenchymal progenitor cells in novel synthetic polymer-hydrogel matrices. Tissue Eng. 9(4), 689-702 (2003)

N. Garcia-Giralt, R. Izquierdo et al., A porous PCL scaffold promotes the human chondrocytes redifferentiation and hyaline-specific extracellular matrix protein synthesis. J Biomed Mater Res A 85A(4), 1082-1089 (2008)

G. Giavaresi, M. Tschon et al., New polymers for drug delivery systems in orthopaedics: in vivo biocompatibility evaluation. Biomed. Pharmacother. 58(8), 411-417 (2004)

D.T. Hartong, E.L. Berson et al., Retinitis pigmentosa. Lancet 368 (9549), 1795-1809 (2006)

J. Holmbom, A. Sodergard et al., Long-term evaluation of porous poly (epsilon-caprolactone-co-L-lactide) as a bone-filling material. J Biomed Mater Res A 75A(2), 308-315 (2005)

Q. Huang, D.W. Hutmacher et al., In vivo mesenchymal cell recruitment by a scaffold loaded with transforming growth factor beta 1 and the potential for in situ chondrogenesis. Tissue Eng 8 (3), 469-482 (2002)

N.W. Karuri, P.F. Nealey et al., Structural organization of the cytoskeleton in SV40 human corneal epithelial cells cultured on nano- and microscale grooves. Scanning 30(5), 405-13 (2008)

H. Klassen, J.F. Kiilgaard et al., Progenitor cells from the porcine neural retina express photoreceptor markers after transplantation to the subretinal space of allorecipients. Stem Cells 25(5), 12221230 (2007)

H. Klassen, D.S. Sakaguchi et al., Stem cells and retinal repair. Prog. Retin. Eye Res. 23(2), 149-181 (2004a)

H.J. Klassen, T.F. Ng et al., Multipotent retinal progenitors express developmental markers, differentiate into retinal neurons, and preserve light-mediated behavior. Invest Ophthalmol Vis Sci 45 (11), 4167-73 (2004b)

A.D. Kulkarni, B.D. Kuppermann, Wet age-related macular degeneration. Adv. Drug Deliv. Rev. 57(14), 1994-2009 (2005)

E.B. Lavik, H. Klassen et al., Fabrication of degradable polymer scaffolds to direct the integration and differentiation of retinal progenitors. Biomaterials 26(16), 3187-96 (2005)

K.J. Lowry, K.R. Hamson et al., Polycaprolactone/glass bioabsorbable implant in a rabbit humerus fracture model. J Biomed Mater Res 36(4), 536-541 (1997)

R.E. MacLaren, R.A. Pearson et al., Retinal repair by transplantation of photoreceptor precursors. Nature 444(7116), 203-207 (2006)

G. Marletta, G. Ciapetti et al., Improved osteogenic differentiation of human marrow stromal cells cultured on ion-induced chemically structured poly-epsilon-caprolactone. Biomaterials 28(6), 11321140 (2007)
J.S. Meyer, M.L. Katz et al., Embryonic stem cell-derived neural progenitors incorporate into degenerating retina and enhance survival of host photoreceptors. Stem Cells 24(2), 274-283 (2006)

D. Motlagh, S.E. Senyo et al., Microtextured substrata alter gene expression, protein localization and the shape of cardiac myocytes. Biomaterials 24(14), 2463-76 (2003)

W.L. Neeley, S. Redenti et al., A microfabricated scaffold for retinal progenitor cell grafting. Biomaterials 29(4), 418-426 (2008)

M. Okabe, M. Ikawa et al., 'Green mice' as a source of ubiquitous green cells. Febs Letters 407(3), 313-319 (1997)

J.B. Recknor, J.C. Recknor et al., Oriented astroglial cell growth on micropatterned polystyrene substrates. Biomaterials 25(14), 2753-67 (2004)

J.B. Recknor, D.S. Sakaguchi et al., Directed growth and selective differentiation of neural progenitor cells on micropatterned polymer substrates. Biomaterials 27(22), 4098-4108 (2006)

S. Redenti, W.L. Neeley et al., Engineering retinal progenitor cell and scrollable poly(glycerol-sebacate) composites for expansion and subretinal transplantation. Biomaterials 30(20), 3405-3414 (2009)

S. Redenti, S. Tao et al., Retinal tissue engineering using mouse retinal progenitor cells and a novel biodegradable, thin-film poly (e-caprolactone) nanowire scaffold. Journal of Ocular Biology, Diseases, and Informatics 1(1), 19-29 (2008)

C. Rivolta, D. Sharon et al., Retinitis pigmentosa and allied diseases: numerous diseases, genes, and inheritance patterns. Hum Mol Genet 11(10), 1219-1227 (2002)

C.A. Sundback, J.Y. Shyu et al., Biocompatibility analysis of poly (glycerol sebacate) as a nerve guide material. Biomaterials $\mathbf{2 6}$ (27), 5454-5464 (2005)

M. Takahashi, T.D. Palmer et al., Widespread integration and survival of adult-derived neural progenitor cells in the developing optic retina. Mol. Cell. Neurosci 12(6), 340-348 (1998)

Z.G. Tang, N.P. Rhodes et al., "Control of the domain microstructures of PLGA and PCL binary systems - Importance of morphology in controlled drug release.”. Chem. Eng. Res. Des. 85(A7), 1044-1050 (2007)

S. Tao, C. Young et al., Survival, migration and differentiation of retinal progenitor cells transplanted on micro-machined poly (methyl methacrylate) scaffolds to the subretinal space. Lab on a Chip 7(6), 695-701 (2007)

R.G. Thakar, M.G. Chown et al., Contractility-dependent modulation of cell proliferation and adhesion by microscale topographical cues. Small 4(9), 1416-24 (2008)

M. Tomita, E. Lavik et al., Biodegradable polymer composite grafts promote the survival and differentiation of retinal progenitor cells. Stem Cells 23(10), 1579-1588 (2005)

H. Wang, S.F. Chen et al., Capillary differentiation of endothelial cells on microgrooved surfaces. Journal of Physical Chemistry C 111 (40), 14602-14606 (2007)

K. Warfvinge, J.F. Kiilgaard et al., Retinal progenitor cell xenografts to the pig retina - Morphologic integration and cytochemical differentiation. Arch Ophthalmol 123(10), 1385-1393 (2005)

G.M. Whitesides, E. Ostuni et al., Soft lithography in biology and biochemistry. Annu Rev Biomed Eng 3, 335-73 (2001)

E.K.F. Yim, S.W. Pang et al., Synthetic nanostructures inducing differentiation of human mesenchymal stem cells into neuronal lineage. Exp Cell Res 313(9), 1820-1829 (2007)

E.K.F. Yim, R.M. Reano et al., Nanopattern-induced changes in morphology and motility of smooth muscle cells. Biomaterials $\mathbf{2 6}$ (26), 5405-5413 (2005)

M.J. Young, J. Ray et al., Neuronal differentiation and morphological integration of hippocampal progenitor cells transplanted to the retina of immature mature dystrophic rats. Mol. Cell. Neurosci. 16(3), 197-205 (2000) 